\title{
AccBou-Tunisia'08.tex \\ ON THE CENTRAL EXTENSIONS OF THE HEISENBERG ALGEBRA
}

\author{
LUIGI ACCARDI AND ANDREAS BOUKAS
}

\begin{abstract}
We describe the nontrivial central extensions $C E(H e i s)$ of the Heisenberg algebra and their representation as sub-algebras of the Schroedinger algebra. We also present the characteristic and moment generating functions of the random variable corresponding to the self-adjoint sum of the generators of $C E($ Heis $)$.
\end{abstract}

\section{Central extensions of Lie algebras}

In the applications of Lie algebras to physical systems the symmetries of the system are frequently described at the level of classical mechanics by some Lie algebra $L$, and in the quantum theoretic description by $L$ plus some extra, constant, not arbitrary terms which are interpreted as the eigenvalues of some new operators $K^{i}$ which have constant eigenvalue on any irreducible module of $L$ (by Schur's lemma the $K^{i}$ must commute with all elements of $L$ ). The new generators $K^{i}$ extend $L$ to a new Lie algebra $\hat{L}$.

In general, given a Lie algebra $L$ with basis $\left\{T^{a} ; a=1,2, \ldots, d\right\}$, by attaching additional generators $\left\{K^{i} ; i=\right.$ $1,2, \ldots, l\}$ such that

$$
\left[K^{i}, K^{j}\right]=\left[T^{a}, K^{j}\right]=0
$$

we obtain an $l$-dimensional central extension $\hat{L}$ of $L$ with Lie brackets

$$
\left[T^{a}, T^{b}\right]=\sum_{c=1}^{d} f_{c}^{a b} T^{c}+\sum_{i=1}^{l} g_{i}^{a b} K^{i}
$$

where $f_{c}^{a b}$ are the structure constants of $L$ in the basis $\left\{T^{a} ; a=1,2, \ldots, d\right\}$. If through a constant redefinition of the generators $\left\{T^{a} ; a=1,2, \ldots, d\right\}$ (i.e. if $\hat{L}$ is the direct sum of $L$ and an Abelian algebra) the commutation relations of $\hat{L}$ reduce to those of $L$ then the central extension is trivial.

A basis independent (or cocycle) definition of an one-dimensional (i.e. having only one central generator) central extension can be given as follows:

If $L$ and $\hat{L}$ are two complex Lie algebras, we say that $\hat{L}$ is an one-dimensional central extension of $L$ with central element $E$ if

$$
\left[l_{1}, l_{2}\right]_{\hat{L}}=\left[l_{1}, l_{2}\right]_{L}+\phi\left(l_{1}, l_{2}\right) E ; \quad\left[l_{1}, E\right]_{\hat{L}}=0
$$

for all $l_{1}, l_{2} \in L$, where $[\cdot, \cdot]_{\hat{L}}$ and $[\cdot, \cdot]_{L}$ are the Lie brackets in $\hat{L}$ and $L$ respectively, and $\phi: L \times L \mapsto C$ is a bilinear form (2-cocycle) on $L$ satisfying the skew-symmetry condition

$$
\phi\left(l_{1}, l_{2}\right)=-\phi\left(l_{2}, l_{1}\right)
$$

Date: January 30, 2009

Key words and phrases. Heisenberg algebra; Schroedinger algebra; Central extension of a Lie algebra; Fock space. 
and the Jacobi identity

$$
\phi\left(\left[l_{1}, l_{2}\right]_{L}, l_{3}\right)+\phi\left(\left[l_{2}, l_{3}\right]_{L}, l_{1}\right)+\phi\left(\left[l_{3}, l_{1}\right]_{L}, l_{2}\right)=0
$$

A central extension is trivial if there exists a linear function $f: L \mapsto \mathbb{C}$ satisfying for all $l_{1}, l_{2} \in L$

$$
\phi\left(l_{1}, l_{2}\right)=f\left(\left[l_{1}, l_{2}\right]_{L}\right)
$$

For more information on central extensions we refer to [3]. Detailed proofs of the material presented in sections $2-5$ below will appear in [1]

\section{Central extensions of the Heisenberg algebra}

The Heisenberg $*$-Lie algebra Heis is the 3 -dimensional Lie algebra with generators $\left\{a^{\dagger}, a, h\right\}$, commutation relations

$$
\left[a, a^{\dagger}\right]_{\text {Heis }}=h \quad ; \quad\left[a, h^{\dagger}\right]_{\text {Heis }}=[h, a]_{\text {Heis }}=0
$$

and involution

$$
\left(a^{\dagger}\right)^{*}=a \quad ; \quad(a)^{*}=a^{\dagger} \quad ; \quad(h)^{*}=h
$$

All 2-cocycles $\phi$ corresponding to a central extension $C E(H e i s)$ of Heis are of the form

$$
\begin{aligned}
\phi\left(a, a^{\dagger}\right) & =\lambda \\
\phi\left(h, a^{\dagger}\right) & =z \\
\phi(a, h) & =\bar{z} \\
\phi(h, h) & =\phi\left(a^{\dagger}, a^{\dagger}\right)=\phi(a, a)=0
\end{aligned}
$$

where $\lambda \in \mathbb{R}$ and $z \in \mathbb{C}$. To see that, let $l_{i}=a_{i} a^{\dagger}+b_{i} a+c_{i} h$ where $a_{i}, b_{i}, c_{i} \in \mathbb{C}$ for all $i \in\{1,2,3\}$, be three elements of Heis. Then

$$
\begin{aligned}
& {\left[l_{1}, l_{2}\right]_{\text {Heis }}=\left(b_{1} a_{2}-a_{1} b_{2}\right) h} \\
& {\left[l_{2}, l_{3}\right]_{\text {Heis }}=\left(b_{2} a_{3}-a_{2} b_{3}\right) h} \\
& {\left[l_{3}, l_{1}\right]_{\text {Heis }}=\left(b_{3} a_{1}-a_{3} b_{1}\right) h}
\end{aligned}
$$

and

$$
\begin{aligned}
\phi\left(\left[l_{1}, l_{2}\right]_{\text {Heis }}, l_{3}\right)= & \left(b_{1} a_{2} a_{3}-a_{1} b_{2} a_{3}\right) \phi\left(h, a^{\dagger}\right) \\
& +\left(b_{1} a_{2} b_{3}-a_{1} b_{2} b_{3}\right) \phi(h, a) \\
\phi\left(\left[l_{2}, l_{3}\right]_{\text {Heis }}, l_{1}\right)= & \left(b_{2} a_{3} a_{1}-a_{2} b_{3} a_{1}\right) \phi\left(h, a^{\dagger}\right) \\
& +\left(b_{2} a_{3} b_{1}-a_{2} b_{3} b_{1}\right) \phi(h, a) \\
\phi\left(\left[l_{3}, l_{1}\right]_{\text {Heis }}, l_{2}\right)= & \left(b_{3} a_{1} a_{2}-a_{3} b_{1} a_{2}\right) \phi\left(h, a^{\dagger}\right) \\
& +\left(b_{3} a_{1} b_{2}-a_{3} b_{1} b_{2}\right) \phi(h, a)
\end{aligned}
$$

and the Jacobi identity (1.5) for $\phi$ reduces to

$$
0 \cdot \phi\left(h, a^{\dagger}\right)+0 \cdot \phi(h, a)=0
$$


which implies that $\phi\left(h, a^{\dagger}\right)$ and $\phi(h, a)$ are arbitrary complex numbers. Since it does not appear in (2.13), $\phi\left(a, a^{\dagger}\right)$ is also an arbitrary complex number. Therefore the, non-zero among generators, $C E(H e i s)$ commutation relations have the form

$$
\begin{aligned}
{\left[a, a^{\dagger}\right]_{C E(\text { Heis })} } & =h+\phi\left(a, a^{\dagger}\right) E \\
{[a, h]_{C E(\text { Heis })} } & =\phi(a, h) E \\
{\left[h, a^{\dagger}\right]_{C E(\text { Heis })} } & =\phi\left(h, a^{\dagger}\right) E
\end{aligned}
$$

where $E$ is the, non-zero, central element. By skew-symmetry

$$
\phi\left(a^{\dagger}, h\right)=-\phi\left(h, a^{\dagger}\right) ; \phi\left(a^{\dagger}, a\right)=-\phi\left(a, a^{\dagger}\right) ; \phi(a, h)=-\phi(h, a)
$$

and

$$
\phi(a, a)=\phi\left(a^{\dagger}, a^{\dagger}\right)=\phi(h, h)=0
$$

By taking the adjoints of (2.14)-(2.16), assuming the involution conditions

$$
\left(a^{\dagger}\right)^{*}=a \quad ; \quad(a)^{*}=a^{\dagger} \quad ; \quad(h)^{*}=h ;(E)^{*}=E
$$

we find that

$$
\phi\left(a, a^{\dagger}\right)=\overline{\phi\left(a, a^{\dagger}\right)}=\lambda \in \mathbb{R}
$$

and

$$
\phi(a, h)=\overline{\phi\left(h, a^{\dagger}\right)}=\bar{z}
$$

where

$$
z=\phi\left(h, a^{\dagger}\right) \in \mathbb{C}
$$

If a central extension $C E(H e i s)$ of Heis is trivial then there exists a linear complex-valued function $f$ defined on Heis such that

$$
\begin{aligned}
f\left(\left[a, a^{\dagger}\right]_{\text {Heis }}\right) & =\lambda \\
f\left([a, h]_{\text {Heis }}\right) & =\bar{z} \\
f\left(\left[h, a^{\dagger}\right]_{\text {Heis }}\right) & =z
\end{aligned}
$$

Since $\left[h, a^{\dagger}\right]_{\text {Heis }}=0$ and (for a linear $f$ ) $f(0)=0$, by (2.25) we conclude that $z=0$.

Conversely, suppose that $z=0$. Define a linear complex-valued function $f$ on Heis by

$$
f\left(z_{1} h+z_{2} a^{\dagger}+z_{3} a\right)=z_{1} \lambda
$$

where $\lambda$ is as above and $z_{1}, z_{2}, z_{3} \in \mathbb{C}$. Then 


$$
\begin{aligned}
f\left(\left[a, a^{\dagger}\right]_{\text {Heis }}\right) & =f\left(1 h+0 a^{\dagger}+0 a\right)=\lambda=\phi\left(a, a^{\dagger}\right) \\
f\left([a, h]_{\text {Heis }}\right) & =f\left(0 h+0 a^{\dagger}+0 a\right)=0=\bar{z}=\phi(a, h) \\
f\left(\left[h, a^{\dagger}\right]_{\text {Heis }}\right) & =f\left(0 h+0 a^{\dagger}+0 a\right)=0=z=\phi\left(h, a^{\dagger}\right)
\end{aligned}
$$

which, by (1.6), implies that the central extension is trivial.

Thus, a central extension of Heis is trivial if and only if $z=0$.

The centrally extended Heisenberg commutation relations (2.14)-(2.16) now have the form

$$
\left[a, a^{\dagger}\right]_{C E(\text { Heis })}=h+\lambda E ;\left[h, a^{\dagger}\right]_{C E(\text { Heis })}=z E ; \quad[a, h]_{H e i s}=\bar{z} E
$$

Renaming $h+\lambda E$ to just $h$ we obtain the equivalent (canonical) $C E$ (Heis) commutation relations

$$
\left[a, a^{\dagger}\right]_{C E(H e i s)}=h ;\left[h, a^{\dagger}\right]_{C E(H e i s)}=z E ;[a, h]_{C E(\text { Heis })}=\bar{z} E
$$

For $z=0$ we recover the Heisenberg commutation relations (2.1). Commutation relations (2.31) define a nilpotent (thus solvable) four-dimensional $*-$ Lie algebra $C E$ (Heis) with generators $a, a^{\dagger}, h$ and $E$. Moreover, if we define $p, q$ and $H$ by

$$
a^{\dagger}=p+i q \quad ; \quad a=p-i q \quad ; \quad H=-i h / 2
$$

then $p, q, E$ are self-adjoint, $H$ is skew-adjoint, and $\{p, q, E, H\}$ are the generators of a real four-dimensional solvable $*$-Lie algebra with central element $E$ and commutation relations

$$
[p, q]=H ;[q, H]=c E ;[H, p]=b E
$$

where $b, c$ are (not simultaneously zero) real numbers given by

$$
c=\frac{\operatorname{Re} z}{2}, b=\frac{\operatorname{Im} z}{2}
$$

Conversely, if $p, q, H, E$ are the generators (with $p, q, E$ self-adjoint and $H$ skew-adjoint) of a real fourdimensional solvable $*$-Lie algebra with central element $E$ and commutation relations (2.33) with $b, c \in \mathbb{R}$ not simultaneously zero, then, defining $z$ by (2.34), the operators $a, a^{\dagger}, h$ defined by $(2.32)$ and $E$ are the generators of the nontrivial central extension $C E$ (Heis) of the Heisenberg algebra defined by (2.31) and (2.19).

The real four-dimensional solvable Lie algebra generated by $\{p, q, E, H\}$ can be identified to the Lie algebra $\eta_{4}$ (one of the fifteen classified real four-dimensional solvable Lie algebras, see for example [4]) with generators $e_{1}, e_{2}, e_{3}, e_{4}$ and (non-zero) commutation relations among generators

$$
\left[e_{4}, e_{1}\right]=e_{2} \quad ; \quad\left[e_{4}, e_{2}\right]=e_{3}
$$

This algebra has been studied by Feinsilver and Schott in [2] 


\section{Representations of $C E($ Heis $)$}

The non-trivial central extensions of $C E$ (Heis) (corresponding to $z \neq 0$ ) can be realized as proper subalgebras of the Schroedinger algebra, i.e. the six-dimensional $*$-Lie algebra generated by $b, b^{\dagger}, b^{2}, b^{\dagger^{2}}, b^{\dagger} b$ and 1 where $b^{\dagger}, b$ and 1 are the generators of a Boson Heisenberg algebra with

$$
\left[b, b^{\dagger}\right]=1 \quad ; \quad\left(b^{\dagger}\right)^{*}=b
$$

More precisely,

(i) If $z \in \mathbb{C}$ with $R e z \neq 0$, then for arbitrary $\rho, r \in \mathbb{R}$ with $r \neq 0$, letting

$$
\begin{aligned}
a & =\left(\frac{4 \rho \operatorname{Im} z-r^{2}}{4 \operatorname{Re} z}+i \rho\right)\left(b-b^{\dagger}\right)^{2}-\frac{i \bar{z}}{2 r}\left(b+b^{\dagger}\right) \\
a^{\dagger} & =\left(\frac{4 \rho \operatorname{Im} z-r^{2}}{4 \operatorname{Re} z}-i \rho\right)\left(b-b^{\dagger}\right)^{2}+\frac{i z}{2 r}\left(b+b^{\dagger}\right)
\end{aligned}
$$

and

$$
h=i r\left(b^{\dagger}-b\right)
$$

we find that the quadruple $\left\{a^{+}, a, h, E=1\right\}$ satisfies commutation relations (2.31) and duality relations (2.19) of $C E($ Heis $)$.

(ii) If $z \in \mathbb{C}$ with $\operatorname{Re} z=0$, then for arbitrary $\rho, r \in \mathbb{R}$ with $r \neq 0$, letting

$$
\begin{aligned}
a & =\left(\rho-\frac{i \operatorname{Im} z}{16 r^{2}}\right)\left(b-b^{\dagger}\right)^{2}+r\left(b+b^{\dagger}\right) \\
a^{\dagger} & =\left(\rho+\frac{i \operatorname{Im} z}{16 r^{2}}\right)\left(b-b^{\dagger}\right)^{2}+r\left(b+b^{\dagger}\right)
\end{aligned}
$$

and

$$
h=\frac{i \operatorname{Im} z}{2 r}\left(b^{\dagger}-b\right)
$$

we find that the quadruple $\left\{a^{+}, a, h, E=1\right\}$ satisfies commutation relations (2.31) and duality relations (2.19) of $C E($ Heis $)$.

Using the fact that for non-negative integers $n, k$

$$
b^{\dagger^{n}} b^{k} y(\xi)=\left.\xi^{k} \frac{\partial^{n}}{\partial \epsilon^{n}}\right|_{\epsilon=0} y(\xi+\epsilon)
$$

where, for $\xi \in \mathbb{C}, y(\xi)=e^{\xi b}$ we may represent $C E(H e i s)$ on the Heisenberg Fock space $\mathcal{F}$ defined as the Hilbert space completion of the linear span of the exponential vectors $\{y(\xi) ; \xi \in \mathbb{C}\}$ with respect to the inner product

$$
\langle y(\xi), y(\mu)\rangle=e^{\bar{\xi} \mu}
$$

We have that:

(i) If $z \in \mathbb{C}$ with $\operatorname{Re} z \neq 0$ then 


$$
\begin{gathered}
a y(\xi)=\left(\left(\frac{4 \rho I m z-r^{2}}{4 \operatorname{Re} z}+i \rho\right)\left(\xi^{2}-1\right)-\frac{i \bar{z}}{2 r} \xi\right) y(\xi) \\
+\left(\left.\left(\frac{4 \rho I m z-r^{2}}{4 \operatorname{Re} z}+i \rho\right) \frac{\partial^{2}}{\partial \epsilon^{2}}\right|_{\epsilon=0}-\left.\left(\left(\frac{4 \rho I m z-r^{2}}{4 \operatorname{Re} z}+i \rho\right) 2 \xi+\frac{i \bar{z}}{2 r}\right) \frac{\partial}{\partial \epsilon}\right|_{\epsilon=0}\right) y(\xi+\epsilon) \\
a^{\dagger} y(\xi)=\left(\left(\frac{4 \rho I m z-r^{2}}{4 \operatorname{Re} z}-i \rho\right)\left(\xi^{2}-1\right)+\frac{i z}{2 r} \xi\right) y(\xi) \\
+\left(\left.\left(\frac{4 \rho I m z-r^{2}}{4 \operatorname{Re} z}-i \rho\right) \frac{\partial^{2}}{\partial \epsilon^{2}}\right|_{\epsilon=0}-\left.\left(\left(\frac{4 \rho I m z-r^{2}}{4 \operatorname{Re} z}-i \rho\right) 2 \xi-\frac{i z}{2 r}\right) \frac{\partial}{\partial \epsilon}\right|_{\epsilon=0}\right) y(\xi+\epsilon) \\
h y(\xi)=i r\left(\left.\frac{\partial}{\partial \epsilon}\right|_{\epsilon=0} y(\xi+\epsilon)-\xi y(\xi)\right)
\end{gathered}
$$

and

$$
E y(\xi)=y(\xi)
$$

(ii) If $z \in \mathbb{C}$ with $\operatorname{Re} z=0$ then

$$
\begin{gathered}
a y(\xi)=\left(\left(\rho-\frac{i \operatorname{Im} z}{16 r^{2}}\right)\left(\xi^{2}-1\right)+r \xi\right) y(\xi) \\
+\left(\left.\left(\rho-\frac{i I m z}{16 r^{2}}\right) \frac{\partial^{2}}{\partial \epsilon^{2}}\right|_{\epsilon=0}+\left.\left(r-\left(\rho-\frac{i \operatorname{Im} z}{16 r^{2}}\right) 2 \xi\right) \frac{\partial}{\partial \epsilon}\right|_{\epsilon=0}\right) y(\xi+\epsilon) \\
a^{\dagger} y(\xi)=\left(\left(\rho+\frac{i \operatorname{Im} z}{16 r^{2}}\right)\left(\xi^{2}-1\right)+r \xi\right) y(\xi) \\
+\left(\left.\left(\rho+\frac{i I m z}{16 r^{2}}\right) \frac{\partial^{2}}{\partial \epsilon^{2}}\right|_{\epsilon=0}+\left.\left(r-\left(\rho+\frac{i I m z}{16 r^{2}}\right) 2 \xi\right) \frac{\partial}{\partial \epsilon}\right|_{\epsilon=0}\right) y(\xi+\epsilon) \\
h y(\xi)=\frac{i \operatorname{Im} z}{2 r}\left(\left.\frac{\partial}{\partial \epsilon}\right|_{\epsilon=0} y(\xi+\epsilon)-\xi y(\xi)\right)
\end{gathered}
$$

and

$$
E y(\xi)=y(\xi)
$$

\section{Random Variables associated with $C E$ (Heis)}

Self-adjoint operators $X$ on the Heisenberg Fock space $\mathcal{F}$ correspond to classical random variables with moment generating function $\left\langle\Phi, e^{s X} \Phi\right\rangle$ and characteristic function $\left\langle\Phi, e^{i s X} \Phi\right\rangle$, where $s \in \mathbb{R}$ and $\Phi$ is the Heisenberg Fock space cyclic vacuum vector such that $b \Phi=0$.

Using the splitting (or disentanglement) formula

$$
e^{s\left(L b^{2}+L b^{\dagger^{2}}-2 L b^{\dagger} b-L+M b+N b^{\dagger}\right)} \Phi=e^{w_{1}(s) b^{\dagger^{2}}} e^{w_{2}(s) b^{\dagger}} e^{w_{3}(s)} \Phi
$$

where $L \in \mathbb{R}, M, N \in \mathbb{C}, s \in \mathbb{R}$,

$$
\begin{aligned}
w_{1}(s) & =\frac{L s}{2 L s+1} \\
w_{2}(s) & =\frac{L(M+N) s^{2}+N s}{2 L s+1}
\end{aligned}
$$


and

$$
w_{3}(s)=\frac{(M+N)^{2}\left(L^{2} s^{4}+2 L s^{3}\right)+3 M N s^{2}}{6(2 L s+1)}-\frac{\ln (2 L s+1)}{2}
$$

we find that the moment generating function $M G F_{X}$ of the self-adjoint operator

$$
X=a+a^{\dagger}+h
$$

where $a, a^{\dagger}, h$ are three of the generators of $C E(H e i s)$, is

$$
M G F_{X}(s)=\left\langle\Phi, e^{s\left(a+a^{\dagger}+h\right)} \Phi\right\rangle=(2 L s+1)^{-1 / 2} e^{\frac{(M+N)^{2}\left(L^{2} s^{4}+2 L s^{3}\right)+3 M N s^{2}}{6(2 L s+1)}}
$$

where $s \in \mathbb{R}$ is such that $2 L s+1>0$.

Similarly, the characteristic function of $X$ is

$$
C F_{X}(s)=\left\langle\Phi, e^{i s\left(a+a^{\dagger}+h\right)} \Phi\right\rangle=(2 i L s+1)^{-1 / 2} e^{\frac{(M+N)^{2}\left(L^{2} s^{4}-2 i L s^{3}\right)-3 M N s^{2}}{6(2 i L s+1)}}
$$

In both $M G F_{X}$ and $C F_{X}$, in the notation of section 3:

(i) if $\operatorname{Re} z \neq 0$ then

$$
\begin{aligned}
L & =\frac{4 \rho \operatorname{Im} z-r^{2}}{2 \operatorname{Re} z} \\
M & =-\left(\frac{\operatorname{Im} z}{r}+i r\right) \\
N & =-\left(\frac{\operatorname{Im} z}{r}-i r\right)
\end{aligned}
$$

(ii) if $\operatorname{Re} z=0$ then

$$
\begin{aligned}
L & =2 \rho \\
M & =2 r-i \frac{\operatorname{Im} z}{2 r} \\
N & =2 r+i \frac{\operatorname{Im} z}{2 r}
\end{aligned}
$$

Notice that, if $L=0$ (corresponding to $\rho \operatorname{Im} z>0$ and $r^{2}=4 \rho \operatorname{Im} z$ in the case when $\operatorname{Re} z \neq 0$ and to $\rho=0$ in the case when $\operatorname{Re} z=0)$ then

$$
M G F_{X}(s)=e^{\frac{M N s^{2}}{2}}= \begin{cases}e^{\left(\frac{(I m z)^{2}}{2 r^{2}}+\frac{r^{2}}{2}\right) s^{2}} & \text { if } \operatorname{Re} z \neq 0 \\ e^{\left(2 r^{2}+\frac{(I m z)^{2}}{8 r^{2}}\right) s^{2}} & \text { if } \operatorname{Re} z=0\end{cases}
$$

which means that $X$ is a Gaussian random variable.

For $L \neq 0$ the term $(2 L s+1)^{-1 / 2}$ is the moment generating function of a gamma random variable.

We may also represent $C E(H e i s)$ in terms of two independent CCR copies as follows: 
For $j, k \in\{1,2\}$ let $\left[q_{j}, p_{k}\right]=\frac{i}{2} \delta_{j, k}$ and $\left[q_{j}, q_{k}\right]=\left[p_{j}, p_{k}\right]=0$ with $p_{j}^{*}=p_{j}, q_{j}^{*}=q_{j}$ and $i^{2}=-1$.

(i) If $z \in \mathbb{C}$ with $\operatorname{Re} z \neq 0$ and $\operatorname{Im} z \neq 0$ then

$$
\begin{aligned}
a & =i \operatorname{Re} z q_{1}+\frac{1}{\operatorname{Re} z} p_{1}^{2}-\operatorname{Im} z p_{2}-\frac{i}{\operatorname{Im} z} q_{2}^{2} \\
a^{\dagger} & =-i \operatorname{Re} z q_{1}+\frac{1}{\operatorname{Re} z} p_{1}^{2}-\operatorname{Im} z p_{2}+\frac{i}{\operatorname{Im} z} q_{2}^{2} \\
h & =-2\left(p_{1}+q_{2}\right)
\end{aligned}
$$

and $E=1$ satisfy the commutation relations (2.31) and the duality relations (2.19) of $C E$ (Heis).

(ii) If $z \in \mathbb{C}$ with $\operatorname{Re} z=0$ and $\operatorname{Im} z \neq 0$ then for arbitrary $r \in \mathbb{R}$ and $c \in \mathbb{C}$

$$
\begin{aligned}
a & =c p_{1}^{2}-\operatorname{Im} z p_{2}+\left(r-\frac{i}{\operatorname{Imz}}\right) q_{2}^{2} \\
a^{\dagger} & =\bar{c} p_{1}^{2}-\operatorname{Im} z p_{2}+\left(r+\frac{i}{\operatorname{Imz}}\right) q_{2}^{2} \\
h & =-2 q_{2}
\end{aligned}
$$

and $E=1$ satisfy the commutation relations (2.31) and the duality relations (2.19) of $C E$ (Heis).

(iii) If $z \in \mathbb{C}$ with $\operatorname{Re} z \neq 0$ and $\operatorname{Im} z=0$ then for arbitrary $r \in \mathbb{R}$ and $c \in \mathbb{C}$

$$
\begin{aligned}
a & =i \operatorname{Re} z q_{1}+\left(\frac{1}{\operatorname{Re} z}+i r\right) p_{1}^{2}+c q_{2}^{2} \\
a^{\dagger} & =-i \operatorname{Re} z q_{1}+\left(\frac{1}{\operatorname{Re} z}-i r\right) p_{1}^{2}+\bar{c} q_{2}^{2} \\
h & =-2 p_{1}
\end{aligned}
$$

and $E=1$ satisfy the commutation relations (2.31) and the duality relations (2.19) of $C E$ (Heis).

We may take

$$
q_{1}=\frac{b_{1}+b_{1}^{\dagger}}{2} ; p_{1}=\frac{i\left(b_{1}^{\dagger}-b_{1}\right)}{2}
$$

and

$$
q_{2}=\frac{b_{2}+b_{2}^{\dagger}}{2} ; p_{2}=\frac{i\left(b_{2}^{\dagger}-b_{2}\right)}{2}
$$

where

$$
\left[b_{1}, b_{1}^{\dagger}\right]=\left[b_{2}, b_{2}^{\dagger}\right]=1
$$

and

$$
\left[b_{1}^{\dagger}, b_{2}^{\dagger}\right]=\left[b_{1}, b_{2}\right]=\left[b_{1}, b_{2}^{\dagger}\right]=\left[b_{1}^{\dagger}, b_{2}\right]=0
$$

In that case, $M G F_{X}$ would be the product of the moment generating functions of two independent random variables defined in terms of the generators of two mutually commuting Schroedinger algebras. 


\section{The Centrally eXtended Heisenberg Group}

For $u, v, w, y \in \mathbb{C}$ define

$$
g(u, v, w, y)=e^{u a^{\dagger}} e^{v h} e^{w a} e^{y E}
$$

The family of operators of the form (5.1) is a group with group law given by

$$
\begin{gathered}
g(\alpha, \beta, \gamma, \delta) g(A, B, C, D)= \\
=g\left(\alpha+A, \beta+B+\gamma A, \gamma+C,\left(\frac{\gamma A^{2}}{2}+\beta A\right) z+\left(\frac{\gamma^{2} A}{2}+\gamma B\right) \bar{z}+\delta+D\right)
\end{gathered}
$$

Restricting to $u, v, w \in \mathbb{R}$ and $y \in \mathbb{C}$ we obtain the centrally extended Heisenberg group $\mathbb{R}^{3} \times \mathbb{C}$ endowed with the composition law:

$$
\begin{gathered}
(\alpha, \beta, \gamma, \delta)(A, B, C, D)= \\
\left(\alpha+A, \beta+B+\gamma A, \gamma+C,\left(\frac{\gamma A^{2}}{2}+\beta A\right) z+\left(\frac{\gamma^{2} A}{2}+\gamma B\right) \bar{z}+\delta+D\right)
\end{gathered}
$$

\section{REFERENCES}

[1] Accardi, L., Boukas, A.: Central extensions of the Heisenberg algebra, submitted (2008)

[2] Feinsilver, P. J., Schott, R.: Differential relations and recurrence formulas for representations of Lie groups, Stud. Appl. Math., 96 (1996), no. 4, 387-406.

[3] Fuchs, J., Schweigert C. : Symmetries, Lie Algebras and Representations (A graduate course for physicists), Cambridge Monographs on Mathematical Physics, Cambridge University Press, 1997

[4] Ovando, G.: Four dimensional symplectic Lie algebras, Beitrage Algebra Geom. 47 (2)(2006) 419-434

Centro Vito Volterra, Università di Roma Tor Vergata, via Columbia 2, 00133 Roma, Italy

E-mail address: accardi@volterra.mat.uniroma2.it

$U R L:$ http://volterra.mat. uniroma2.it

Department of Mathematics and Natural Sciences, American College of Greece, Aghia Paraskevi, Athens 15342, Greece

E-mail address: andreasboukas@acgmail.gr 